\title{
Prediction of longer-term outcome of treatment-resistant depression in tertiary care
}

\author{
Abebaw Fekadu, Lena J. Rane, Sarah C. Wooderson, Kalypso Markopoulou, Lucia Poon \\ and Anthony J. Cleare
}

\section{Background}

Systematic studies on the outcome of treatment-resistant depression are scarce.

\section{Aims}

To describe the longer-term outcome and predictors of outcome in treatment-resistant depression.

\section{Method}

Out of 150 patients approached, 118 participants with confirmed treatment-resistant depression (unipolar, $n=77$; bipolar, $n=27$; secondary, $n=14$ ) treated in a specialist in-patient centre were followed-up for between 8 and 84 months (mean $=39$, s.d. $=22$ )

\section{Results}

The majority of participants attained full remission (60.2\%), most of whom (48.3\% of total sample) showed sustained recovery (full remission for at least 6 months). A substantial minority had persistent subsyndromal depression (19.5\%) or persistent depressive episode (20.3\%). Diagnosis of bipolar treatment-resistant depression and poorer social support were associated with early relapse, whereas strong social support, higher educational status and milder level of treatment resistance measured with the Maudsley Staging Method were associated with achieving quicker remission. Exploratory analysis of treatment found positive associations between treatment with a monoamine oxidase inhibitor
(MAOI) in unipolar treatment-resistant depression and attaining remission at discharge and at final follow-up, and duloxetine use predicted attainment of remission at final follow-up.

\section{Conclusions}

Although many patients with treatment-resistant depression experience persistent symptomatology even after intensive, specialist treatment, most can achieve remission. The choice of treatment and presence of good social support may affect remission rates, whereas those with low social support and a bipolar diathesis should be considered at higher risk of early relapse. We suggest that future work to improve the longterm outcome in this disabling form of depression might focus on social interventions to improve support, and the role of neglected pharmacological interventions such as MAOIS.

\section{Declaration of interest}

A.J.C. has within the past 9 years: received honoraria for consulting and/or speaking from Eli Lilly, UCB Pharma, Merck, Organon, Pfizer and Cyberonics; received unrestricted research grant support from GlaxosmithKline; and has written medico-legal reports on patients with depression for court proceedings instructed by both claimants and defendants.
Although treatment-resistant depression is a heterogeneous condition, it is typically defined as a depressive disorder that fails to show satisfactory response (optimally remission) to at least one adequately given antidepressant medication. ${ }^{1,2}$ It is rightly regarded as a significant clinical and public health challenge ${ }^{3}$ owing to its common occurrence, ${ }^{4,5}$ association with serious complications such as self-harm and suicide, and higher level of comorbidity and service utilisation. ${ }^{6-10}$

Treatment-resistant depression has attracted an increasing level of research interest in the past two decades. However, virtually no two studies have defined treatment-resistant depression or its outcomes consistently. ${ }^{11}$ Furthermore, other than in the context of acute treatment trials, there are no systematic reports on the predictors of medium- and longer-term outcomes. ${ }^{11}$ Understanding factors associated with outcome is important in order to better understand the aetiology of the disorder, and to identify potential targets for therapeutic intervention. In this report we describe the findings from a study of the longer-term outcome of treatment-resistant depression, together with the clinical and psychosocial factors that may be associated with outcome, in a well-characterised sample of patients with treatment-resistant depression. Specifically, we aimed to determine the factors associated with remission and recovery, and with illness persistence. The sample was drawn from a tertiary in-patient service in the UK. Because such patients may have a number of pathways to treatment-resistant depressive symptoms, and to maintain a naturalistic and generalisable sample, we included patients with a bipolar diathesis as well as patients in whom depression occurred in the context of another psychiatric disorder. This is particularly relevant given the recent findings that bipolar symptomatology is especially common in those with treatment-resistant depression. ${ }^{12}$

\section{Method}

\section{Design}

The study was a prospective follow-up of patients discharged from a tertiary unit. Follow-up data were collected using both longitudinal and cross-sectional assessments. The report presented here relies on longitudinal assessments using the Longitudinal Interval Follow-up Evaluation (LIFE) chart and Psychiatric Status Rating (PSR). ${ }^{13}$

\section{Participants}

The cohort consisted of patients discharged from a tertiary unit for treatment-resistant mood disorders in the UK. Treatmentresistant depression was defined using the Maudsley Staging Method (MSM), ${ }^{2}$ a multidimensional tool that incorporates treatment failure (antidepressants, augmentations and electroconvulsive therapy (ECT)), duration and severity of depression to grade level of treatment resistance (Table 1). ${ }^{2,14}$ The mean 
Table 1 summary of scoring system and domain components of the Maudsley Staging Method

Score range

Domains

Antidepressants $\quad 1-5$

Failure of augmentations $\quad 0-1$

Failure of electroconvulsive therapy 0-1

Chronicity $1-3$

Severity 1-5

Total score

1-5

Severity categories

Mild

Moderate

3-6

Severe

$7-10$

$11-15$

severity of the level of resistance measured using the MSM was 10.1 (s.d. = 2.2), suggesting that most participants had moderately severe or severe treatment-resistant depression. The MSM was developed based on theoretical considerations, and the staging method has shown good predictive validity for both short-term and longer-term outcomes. ${ }^{2,15}$ As the MSM incorporates failure of antidepressants and of augmentation strategies and mood stabilisers, we have used it for staging bipolar treatment-resistant depression as well, particularly as there is as yet no widely accepted differential definition of treatment-resistant bipolar depression. We also note accumulating evidence that a bipolar diathesis may be more common in treatment-resistant depression, and that treatment non-response is more common in bipolar depression. ${ }^{12}$ In order to maximise diagnostic accuracy, all patients undertook a detailed diagnostic assessment process over at least 4 weeks as described previously. ${ }^{16}$ Follow-up assessment took place a minimum of 1 year after discharge from hospital except in individuals who for practical reasons were followed up 8-11 months post-discharge.

\section{Outcome definitions}

The main outcomes of interest were remission, recovery, relapse and persistence of depressive episode (Table 2). These outcomes were drawn from the PSR scores and were consistent with the operational criteria suggested in previous outcome studies of depressive disorders. ${ }^{17,18}$ Remission was defined as maintaining asymptomatic or nearly asymptomatic state for a minimum of 1 month and was considered from the point of discharge. Recovery from an episode was defined by maintaining remission for at least 6 consecutive months at any stage of follow-up. A more stringent criterion defines recovery as achieving continuous remission in the past 6 months of follow-up. ${ }^{19}$ For an episode, a patient fulfils diagnostic criteria for a depressive episode according to ICD-10. ${ }^{20}$ For a persistent episode, the depressive episode continues throughout the follow-up period. Relapse assumes the participant has developed an episode lasting at least 1 month after attaining full or partial remission. Mortality during this period was also included as a separate adverse outcome.

\section{Measurement of outcome}

The LIFE chart was administered once at the end of the follow-up period to rate the monthly clinical status of participants using all available information, including data from clinical records and informants. The LIFE allows the weekly or monthly symptomatic state of a patient to be rated retrospectively at follow-up intervals of 6 months or longer (up to 96 months). ${ }^{13}$ Symptoms are ordinarily rated on a six-point scale using the PSR. The PSR ratings are operationally linked to DSM-IV ${ }^{21}$ and ICD-10. ${ }^{20}$ In this study, we used a modified PSR rating as adapted for a UK study. ${ }^{22,23}$ The modification expands the PSR ratings from 6 to 7 scores: a score of 1 or 2 corresponds to remission, a score of 3 or 4 corresponds to response/partial remission with subsyndromal symptomatology, and a score of 5, 6 or 7 corresponds to being in a depressive episode $($ mild $=5$, moderate $=6$, severe $=7$ ). The PSR scores were obtained to characterise the monthly clinical status of patients for the interval between discharge from hospital and final follow-up date.

The LIFE chart was administered by two psychiatrists (A.F. and K.M.). The two raters had good interrater reliability (kappa of 0.9 , $P<0.001$ and Spearman's rho of $0.9, P<0.001$ ). The LIFE chart ratings also showed good convergence with concurrently administered cross-sectional clinical scales (the Beck Depression Inventory ${ }^{24}$ and the 21-item Hamilton Rating Scale for Depression (HRSD) ${ }^{25}$ ) and divergence with functional scales (the Global Assessment of Functioning $^{21}$ and the 12-item Short-Form Health Survey ${ }^{26}$ ).

\section{Predictors}

Based on the outcome literature on depression, three main categories were assessed for predicting relapse and remission: (a) sociodemographic predictors; (b) clinical predictors (severity of prior treatment resistance measured with the MSM; age at onset of depression; symptom severity at discharge; and treatments received during admission); and (c) level of social support and life events. A short self-report questionnaire, the Oslo 3 Support Scale $(\mathrm{OSS})^{27}$ was used to assess general social support. The OSS contains 3-items assessing the number of close confidants, perceived level of concern from others and perceived ease of getting help from neighbours. Based on the raw scores, the scale allows a summary score (range 3-14) or categories of social support (strong $v$. poor) to be generated. To assess life events, we used the List of Threatening Experiences. ${ }^{28}$

The effect on remission of individual psychotropic medications, either switching class of antidepressant or using a first- or secondline choice for treatment-resistant depression as listed in the Maudsley Prescribing Guidelines, ${ }^{29}$ was determined if the medication was given to at least ten patients at adequate doses. The analysis was essentially exploratory because of the relatively small number of patients receiving the individual medications.

\begin{tabular}{|c|c|c|c|}
\hline Outcome & $\begin{array}{l}\text { Psychiatric Status } \\
\text { Rating score }\end{array}$ & ICD-10 status & $\begin{array}{l}\text { Minimum duration, } \\
\text { months }\end{array}$ \\
\hline Remission & $1-2$ & Asymptomatic & 1 \\
\hline Partial remission (response/subthreshold) & $3-4$ & Symptomatic but below episode level & 1 \\
\hline Episode & $5-7$ & Episode criteria & 1 \\
\hline Recovery & $1-2$ & Asymptomatic & 6 \\
\hline Relapse & $5-7$ & Episode criteria & 1 \\
\hline
\end{tabular}


However, despite the limitation of the sample size and the fact that this was not a treatment trial, any supportive evidence regarding effective intervention in this difficult-to-treat diagnostic subgroup is useful.

\section{Data management}

Data were entered in to the Statistical Package for Social Sciences version 15 for Windows. Analysis used SPSS supplemented with STATA, version 11 for Windows (Statacorp). Although some studies in chronic depression and treatment-resistant depression report on pooled data, ${ }^{18,30}$ we stratified analysis according to discharge diagnosis (unipolar, bipolar and secondary treatmentresistant depression ${ }^{31}$ ) in addition to presenting results on pooled data. The term 'secondary' was used in the chronological and not necessarily in the aetiological sense; for example, when treatmentresistant depression developed in the context of an obsessivecompulsive disorder or personality disorder that had started first. For the main data analysis, we employed the Cox multivariate model to determine the independent effect of predictive factors on survival status in remission or in depressive episode.

All patients with potential to relapse into a full episode (40 patients discharged in remission and 36 patients in partial remission as well as 19 patients that achieved full or partial remission during follow-up) were included in the survival analysis. The focus of this study was on depression, and we have not included manic or hypomanic relapses in the analysis. Nevertheless, only two patients had hypomanic and two patients had manic relapses during the follow-up period.

All study procedures were approved by the local ethics committee and all participants provided written, informed consent (ethics approval numbers 285/03 and 322/03).

\section{Results}

\section{Sociodemographic and clinical characteristics of cohort}

Of the 150 patients discharged with treatment-resistant depression and approached, $118(78.7 \%)$ were successfully followed-up. Reasons for non-participation in the study were: refused to take part $(n=7)$, not traced because of change of address $(n=8)$, deceased $(n=13)$ and incomplete data $(n=4)$. There was no significant difference in all baseline characteristics between participants and non-participants except for duration of admission, which was longer for participants (median 24.0 weeks, interquartile range (IQR) 25.0 weeks) than non-participants (median 9.5 weeks, IQR $=21.0)(P<0.01)$.

The baseline sociodemographic and clinical details of the 118 included participants are presented in Table 3. Participants had highly treatment-resistant illness, with $65.5 \%$ having had a history of treatment with ECT, and the mean number of prior antidepressant treatments for the admission episode being 5.9 (s.d.=3.5). Additionally, $60 \%$ of the sample had history of a suicide attempt. The majority of the cohort was female, married or stably cohabiting, and unemployed. The mean duration of illness was nearly 20 years, and the median duration of index (admission) episode was 3 years $(\mathrm{IQR}=7)$. At discharge, $33.9 \%$ $(40 / 118)$ were in remission, while $30.5 \%(36 / 118)$ and $35.6 \%$ $(42 / 118)$ were in partial remission and in depressive episode respectively.

Participants were followed up for a mean of 39.1 months (s.d.=22.0), ranging from 8 to 84 months. The mean HRSD-21 for the whole group was 20.5 (s.d.=6.4), higher for unipolar treatment-resistant depression (mean 21.1, s.d.=6.1) and lower for secondary treatment-resistant depression (mean 17.6,

Table 3 Sociodemographic and clinical characteristics of study sample stratified by diagnostic subtype

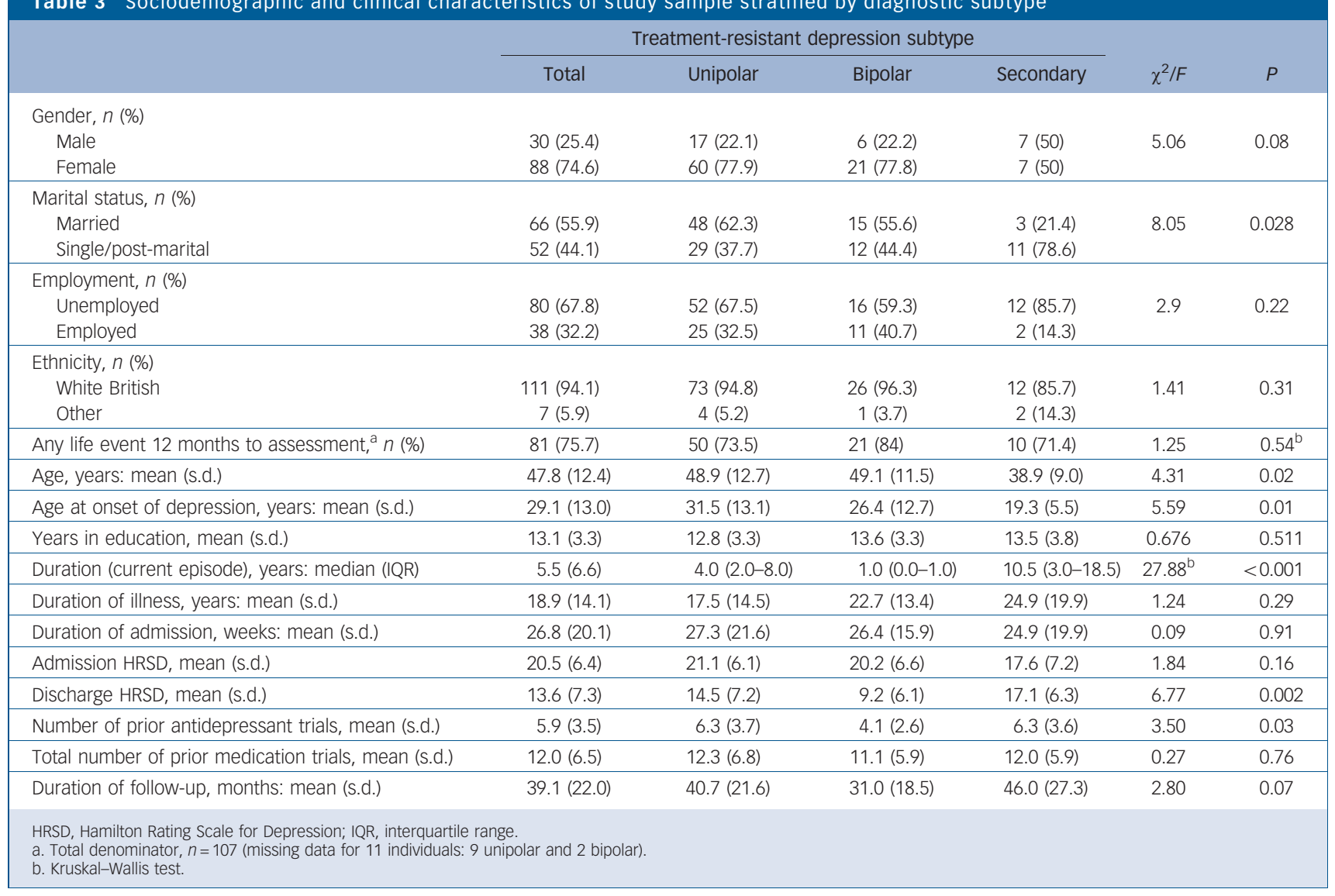


s.d.=7.2), although the differences were not statistically significant (Table 3).

\section{Pharmacological treatments}

The main antidepressant switches were to monoamine oxidase inhibitors (MAOIs) $(n=16)$, duloxetine $(n=22)$ tricyclic antidepressants $(n=28)$, mirtazapine $(n=10)$ and venlafaxine $(n=26)$, whereas augmentation strategies involved the use of lithium $(n=23)$, lamotrigine $(n=50)$, thyroid hormone $(n=16)$ and atypical antipsychotics $(n=25)$. All the major MAOI classes were used (moclobemide, phenelzine, tranylcypromine and isocarboxazid), although phenelzine was the most common. Amitriptyline and imipramine were the two tricyclic antidepressants commonly used. The main atypical antipsychotics used for augmentation were olanzapine, risperidone, quetiapine and aripiprazole. Minimum dose and duration to define an adequate trial was based on the Maudsley Prescribing Guidelines, ${ }^{29}$ supplemented by the Antidepressant History Form ${ }^{32}$ and the British National Formulary. ${ }^{33}$

\section{Main outcomes}

Details are presented in Fig. 1 and Table 4. Overall, 48.3\% $(n=57)$ of the cohort met recovery criteria, and $11.9 \%(n=14)$ had met remission criteria without ever meeting recovery criteria. Of those who met only remission criteria, all except one participant had been in remission for 2 months or longer. Thus, if recovery was defined as achieving remission at least for 2 consecutive months as used in some outcome studies of chronic affective disorders, ${ }^{19}$ the proportion achieving recovery would rise to $59.3 \% \quad(n=70 /$ 118 ). On the other hand, if recovery was defined more stringently as achieving remission in the last 6 months of follow-up, ${ }^{19}$ the figure for recovery drops to $31.4 \% \quad(n=37)$. In terms of unfavourable outcome, $39.8 \%$ had persistent symptomatology throughout the follow-up period, either because of persistent subthreshold/subsyndromal symptoms (19.5\%) or persistent depressive episode $(20.3 \%)$. Nearly two-thirds of the follow-up time $(60.7 \%)$ was spent in a symptomatic state: $36.1 \%$ in episode and $24.6 \%$ in subthreshold state (Table 4 ).

In a stratified comparison between the diagnostic groups, about three-quarters of participants with bipolar treatmentresistant depression $(74.1 \%, n=20)$ had achieved either remission or recovery, whereas the proportion for unipolar $(55.8 \%, n=43)$ and secondary treatment-resistant depression $(57.1 \%, n=8)$ was comparably lower (Fig. 1). However, the only statistically significant difference in outcome between these groups was the higher proportion of participants with persistent episode in the unipolar $(P=0.02)$ and secondary treatment-resistant depression groups $(P=0.01)$ compared with the bipolar treatment-resistant depression group.

Excluding those with persistent episode, 95 participants had the potential to experience relapse; of these, 54.7\% $(n=52)$ experienced a depressive relapse during follow-up.

\section{Mortality}

Mortality is one of the indicators of unfavourable outcome in depression. ${ }^{34}$ Thirteen participants died during follow-up: eight from natural causes (primarily cardiovascular) and five from unnatural causes (suicide, $n=3$; accidental deaths, $n=2$ ). There was a significant trend for association between discharge status and mortality $\chi^{2}=8.03 ; P=0.01$ ). Thus, only two individuals who were discharged in remission died.

\section{Predictors of outcome}

Three main factors were associated with achieving remission during follow-up: severity of treatment-resistant depression measured with the MSM, educational achievement and level of social support. Participants with higher levels of treatment

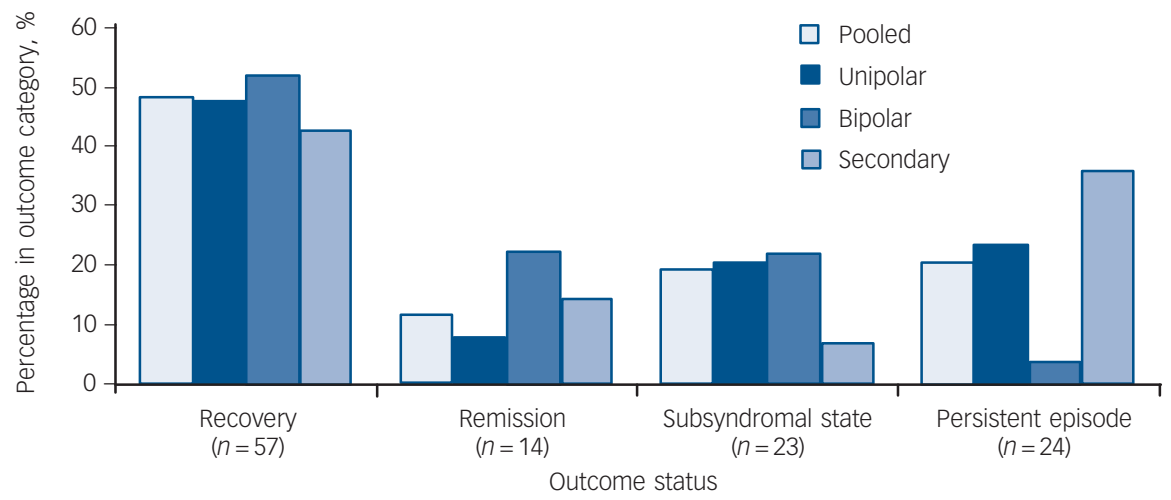

Fig. 1 Proportion of participants in various Iongitudinally defined outcome states stratified by treatment-resistant depression subtype. Total number of participants: $n=118$; unipolar treatment-resistant depression, $n=77$; bipolar treatment-resistant depression, $n=28$; secondary treatment-resistant depression, $n=14$.

Table 4 Percentage time spent in various clinical states stratified by diagnosis and discharge status

\begin{tabular}{|c|c|c|c|}
\hline & \multicolumn{3}{|c|}{ Percentage time spent in: } \\
\hline & Episode & Subthreshold & Remission \\
\hline \multicolumn{4}{|c|}{ Diagnostic subtype of treatment-resistant depression } \\
\hline Unipolar & 39.9 & 35.5 & 24.4 \\
\hline Bipolar & 23.6 & 49.2 & 25.3 \\
\hline Secondary & 39.1 & 34.8 & 24.7 \\
\hline \multicolumn{4}{|c|}{ Clinical status at discharge } \\
\hline Remission & 9.9 & 10.1 & 78.7 \\
\hline Partial remission & 20.5 & 60.0 & 19.3 \\
\hline Episode & 74.1 & 16.2 & 9.0 \\
\hline Overall & 36.1 & 24.6 & 38.5 \\
\hline
\end{tabular}




\begin{tabular}{|c|c|c|c|c|c|}
\hline Variable & $\begin{array}{c}\text { Crude } \\
\text { hazard ratio }\end{array}$ & $95 \% \mathrm{Cl}$ & $\begin{array}{c}\text { Adjusted } \\
\text { hazard ratio }\end{array}$ & $95 \% \mathrm{Cl}$ & $P$ \\
\hline Gender & 0.95 & $0.40-2.24$ & 0.62 & $0.25-1.57$ & 0.32 \\
\hline Life event & 1.17 & $0.60-5.08$ & 1.02 & $0.33-3.11$ & 1.00 \\
\hline Age at onset & 1.60 & $0.99-1.00$ & 1.01 & $0.97-1.05$ & 0.70 \\
\hline Years of education & 1.16 & $1.04-1.29$ & 1.17 & $1.01-1.35$ & 0.03 \\
\hline Severity of treatment-resistant depression ${ }^{\mathrm{b}}$ & 0.77 & $0.65-0.92$ & 0.82 & $0.68-0.99$ & 0.04 \\
\hline Psychiatric Status Rating at discharge & 0.90 & $0.68-1.17$ & 0.89 & $0.39-2.03$ & 0.78 \\
\hline Social support & 1.81 & $1.17-2.81$ & 1.76 & $1.07-2.89$ & 0.03 \\
\hline
\end{tabular}

resistance were less likely to achieve remission, i.e. had more persistent illness (hazard ratio $(\mathrm{HR})=0.77,95 \%$ CI $0.68-0.99$; $P=0.04)$. Participants with higher educational achievement (HR $=1.17,95 \%$ CI $1.01-1.35 ; P=0.03)$ and those with strong social support $(\mathrm{HR}=1.76,95 \% \mathrm{CI} 1.07-2.89 ; P=0.03)$ were more likely to achieve quicker remission (Table 5). The two factors independently associated with relapse were poor social support $(\mathrm{HR}=3.55,95 \%$ CI 1.01-12.54; $P=0.05)$ and receiving a diagnosis of bipolar depression ( $\mathrm{HR}=3.39,95 \%$ CI 1.06-10.98). The survival plot is shown in Fig. 2.

Use of MAOIs while an in-patient was independently associated with remission at point of discharge controlling for other treatments, particularly for unipolar treatment-resistant depression (odds ratio $(\mathrm{OR})=6.49,95 \%$ CI 1.63-25.91). Similarly, in-patient use of MAOIs ( $\mathrm{OR}=4.78,95 \%$ CI 1.15-19.85) or duloxetine $(\mathrm{OR}=4.60,95 \% \mathrm{CI} 1.18-17.93)$ was associated with being in full remission at the time of final follow-up. When analysis was stratified by gender, the effect of duloxetine remained significant only among women $(\mathrm{OR}=6.07,95 \%$ CI 1.59-23.17; $P=0.01$ ). Mean doses of MAOIs used were: phenelzine $70 \mathrm{mg}$; moclobemide $650 \mathrm{mg}$; tranylcypromine $32.5 \mathrm{mg}$; and isocarboxazid $40 \mathrm{mg}$. The mean dose of duloxetine was $98.2 \mathrm{mg}$. Number of medications received prior to admission was only marginally

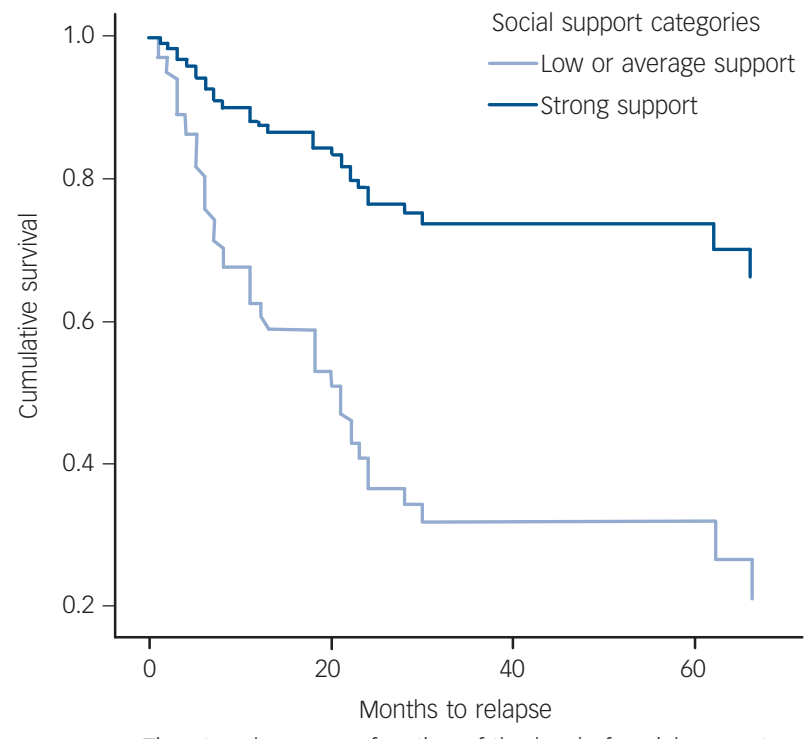

Time to relapse as a function of the level of social support

Fig. 2 Survival curves for time to relapse as a function of the level of social support.

Adjusted for years of education, gender, age at onset, discharge clinical status and diagnosis (hazard ratio $3.55,95 \% \mathrm{Cl} 1.01-12.54 ; P=0.05$ ). but negatively associated with achieving remission $(\mathrm{OR}=0.92$, 95\% CI 0.85-1.00).

\section{Discussion}

Our study is novel in that it takes a longitudinal perspective on the outcome, and predictors of outcome, in well-defined treatmentresistant depression in a naturalistic setting. The main findings of this study relate: first, to a clearer understanding of the expected outcomes in moderate to severe TRD; and second, to indications of potentially modifiable risk factors that may be amenable to intervention or change in order to improve outcome.

\section{Limitations}

Before discussing these results in more detail, some limitations of the study should be highlighted. First, the cohort was identified from a specialist service and is likely to represent the more severe spectrum of illness. Therefore, the results may not fully translate to treatment-resistant depression seen in other settings. Second, the follow-up duration was variable. However, the Cox regression model is suitable for analysis of data with variable duration of follow-up. Third, we could only determine outcomes of 131/150 patients, leaving 19/150 (13\%) unaccounted for. It is possible that these patients had worse outcome, particularly those who refused to take part (7/150). If this is the case, then the proportion with a poor outcome would be slightly higher. Finally, the sample size was modest for a detailed analysis on the various treatmentresistant depression subcategories. However, rather than solely looking at all treatment-resistant depression categories together, as has been done in other studies, we opted also to show the outcomes of the categories separately when applicable so that any differences between unipolar, bipolar and secondary treatmentresistant depression could be observed. Moreover, despite these limitations, to our knowledge, this is the first report on the longer-term outcome of explicitly defined treatment-resistant depression and its predictors using well-operationalised outcome criteria.

\section{General outcome}

The population has a very high level of treatment-resistant illness with 12 prior failed medication trials and a mean MSM score of 10 as well as history of failed trials with ECT in two-thirds of patients, although the admission HRSD severity level was generally in the mild to moderate range according to National Institute for Health and Clinical Excellence guidance. ${ }^{35}$ Admission depression severity may have been influenced by a partial treatment response, which might be expected given the large number of prior treatment exposures. Overall, the proportion achieving remission and 
recovery $(60.2 \%)$ over an average follow-up period of 3 years is substantial. However, a high proportion of participants also experienced persistent episode $(20.3 \%)$ or showed a significant level of persistent and impairing symptomatology (19.5\%). This is particularly so for both the unipolar and the secondary treatment-resistant depression group. Additionally, optimal outcome (remission for at least 6 contiguous months) was achieved in under $50 \%$ of cases. About $10 \%$ of participants died during this follow-up period, most of whom had poorer responses to in-patient treatment. If this latter group was considered also to have unfavourable outcome, the figure for those with the most favourable outcome drops to $43.5 \%$ (57/131). This figure would drop further to $38 \%$ if those who had refused follow-up assessments or were lost to follow-up were again considered to have had worse outcome. This confirms the serious and chronic nature of treatment-resistant depression and the need to improve on current treatments.

There are no directly comparable studies with which we can compare our findings. One of the few well-designed studies on treatment-resistant depression looked at the 1- to 2-year outcome of patients with moderately advanced unipolar and bipolar treatment-resistant depression in a multicentre study setting. ${ }^{30}$ The 1- and 2-year remission rates defined with a self-rated measure were very low $(3.7 \%$ and $7.8 \%$ respectively). However, the study was part of a treatment trial and remission was determined for each visit on the basis of the severity of symptoms in the 7 days prior to the visit rather than longitudinally for the whole follow-up duration. Another follow-up study of 66 patients with well-defined treatment-resistant depression (both unipolar and bipolar) followed up patients for 1.1-59.3 months (mean 29.0). ${ }^{36}$ Outcomes were classified as poor, fair and good, with $71 \%$ categorised as having a good or fair outcome. Despite differences in methodology and follow-up duration, these results may be considered roughly comparable to our findings.

A study conducted as part of the National Institute of Mental Health Collaborative Program on the Psychobiology of Depression-Clinical Studies may be compared with our study in methodology, and to some extent in patient sample. ${ }^{19}$ This report was based on a 5-year follow-up of 129 patients with chronic affective disorders (unipolar, bipolar and schizoaffective disorders), and employed the LIFE chart method. In this study, $77 \%$ of patients followed-up recovered (defined as achieving remission for at least 2 months). When recovery was defined more stringently (achieving remission for the last 6 months of follow-up), about $48 \%$ meet recovery criteria. ${ }^{19}$ When our outcome criteria were modified to fit the US study, the outcome in our sample is somewhat worse, in terms of both the proportion of patients meeting general recovery criteria $(59 \%$ v. $77 \%)$ and more stringently defined recovery criteria $(34.1 \%$ v. $48 \%)$. One explanation could be the shorter duration of follow-up in our sample, since there is a higher chance of recovery with longer follow-up. It could also be due to the inclusion of patients with more severely treatment-resistant illness in our study, because the US study was a follow-up of patients with chronic depression rather than explicitly defined treatment-resistant depression. The main advantage of our report compared with the US study is that our study deals with a poorly researched patient group (those with treatment-resistant depression) and the details are stratified by diagnostic group rather than based solely on analysis of pooled data.

\section{Prediction of outcome}

The main predictors of outcome, specifically predictors of remission and factors predicting early relapse, are consistent with what has been previously reported for depression in general. For example, several studies have reported that social support predicts recovery or treatment response, particularly in the short term. ${ }^{37-39}$ Severity of treatment resistance measured with the MSM was also an important predictor of failure to achieve remission or illness persistence. $^{15}$

Our results also show cross-sectional association between certain medication groups (MAOIs and duloxetine) and better outcome. The use of MAOIs is one of the recommended strategies for managing treatment-resistant depression; studies from over two decades ago had indicated the usefulness of MAOIs in nonresponsive depression ${ }^{40}$ or depression with specific symptom profiles. ${ }^{41}$ However, it occurs only as a third-line option (in combination with a tricyclic antidepressant) in the widely used Maudsley Prescribing Guidelines. ${ }^{29}$ We suggest that our findings act as a reminder that MAOIs have a place in the management of treatment-resistant depression, and require more systematic investigation.

\section{Clinical implications}

In a potentially chronic and relapsing condition such as treatment-resistant depression, studies looking at longitudinal outcome are particularly relevant. The present study represents one of the very few such studies, and confirms the often poor long-term outlook and the need for new treatment approaches to improve outcomes. This is underlined by the association between increasing symptom severity and mortality. Nevertheless, most patients do experience sustained periods of remission. Importantly, we have found that social support influences the long-term outcome of treatment-resistant depression, suggesting that this could be a suitable target for therapeutic intervention. ${ }^{42}$ There are also suggestions that patients with treatment-resistant depression and a bipolar diathesis may have different long-term trajectories, an area that requires further study using larger sample sizes. Finally, the study highlights the need to further investigate the role of certain medications, particularly MAOIs, in the management of treatment-resistant depression.

Abebaw Fekadu, MD, PhD, MRCPsych, Department of Psychiatry, School of Medicine, College of Health Sciences, Addis Ababa University, Addis Ababa, Ethiopia, and Division of Psychological Medicine, Institute of Psychiatry, Kings College London, UK; Lena J. Rane, MBBS, MRCPsych, Sarah C. Wooderson, PhD, Kalypso Markopoulou, MD, MRCPsych, Division of Psychological Medicine, Institute of Psychiatry, Kings College London, UK; Lucia Poon, RMN, Affective Disorder Unit Bethlem Royal Hospital, South London and Maudsley NHS Foundation Trust, UK; Anthony J. Cleare, MBBS, MRCPsych, PhD, Division of Psychological Medicine, Institute of Psychiatry, Kings College London, and Affective Disorder Unit, Bethlem Royal Hospital, South London and Maudsley NHS Foundation Trust, UK

Correspondence: Abebaw Fekadu, Department of Psychiatry, School of Medicine, College of Health Sciences, Addis Ababa University, PO Box 9086, Addis Ababa, Ethiopia. Email: abe.wassie@kcl.ac.uk

First received 9 Sep 2011, final revision 15 Jun 2012, accepted 23 Jul 2012

\section{Funding}

This research was supported by the NIHR Biomedical Research Centre at the South London and Maudsley NHS Foundation Trust and the Institute of Psychiatry (King's College London).

\section{References}

1 Thase ME, Rush AJ. When at first you don't succeed: sequential strategies for antidepressant nonresponders. J Clin Psychiatry 1997; 58: 23-29.

2 Fekadu A, Wooderson S, Donaldson C, Markopoulou K, Masterson B, Poon L, et al. A multidimensional tool to quantify treatment resistance in depression: the Maudsley staging method. J Clin Psychiatry 2009; 70: 177-84.

3 Greden JF. The burden of disease for treatment-resistant depression. J Clin Psychiatry 2001; 62 (suppl 16): 26-31. 
4 Souery D, Papakostas GI,Trivedi MH. Treatment-resistant depression. J Clin Psychiatry 2006; 67 (suppl 6): 16-22.

5 Trivedi MH, Rush AJ, Wisniewski SR, Nierenberg AA, Warden D, Ritz L, et al Evaluation of outcomes with citalopram for depression using measurementbased care in $\mathrm{STAR}^{\star} \mathrm{D}$ : implications for clinical practice. Am J Psychiatry 2006; 163: 28-40.

6 Fava M. Diagnosis and definition of treatment-resistant depression. Biol Psychiatry 2003; 53: 649-59.

7 Keller MB. Issues in treatment-resistant depression. J Clin Psychiatry 2005; 66 (suppl 8): 5-12.

8 Kornstein SG, Schneider RK. Clinical features of treatment-resistant depression. J Clin Psychiatry 2001; 62 (suppl 16): 18-25.

9 Nelsen MR, Dunner DL. Clinical and differential diagnostic aspects of treatment-resistant depression. J Psychiatr Res 1995; 29: 43-50.

10 Sackeim HA. The definition and meaning of treatment-resistant depression. J Clin Psychiatry 2001; 62 (suppl 16): 10-7.

11 Fekadu A, Wooderson SC, Markopoulo K, Donaldson C, Papadopoulos A, Cleare AJ. What happens to patients with treatment-resistant depression? A systematic review of medium to long term outcome studies. J Affect Disord 2009; 116: 4-11.

12 Keller MB, Lavori PW, Friedman B, Nielsen E, Endicott J, McDonald-Scott P et al. The longitudinal interval follow-up evaluation: a comprehensive method for assessing outcome in prospective longitudinal studies. Arch Gen Psychiatry 1987; 44: 540-8.

13 Ruhé HG, van Rooijen G, Spijker J, Peeters FPML, Schene AH. Staging methods for treatment resistant depression. A systematic review. J Affect Disord 2012: 137: 35-45.

14 Fekadu A, Wooderson SC, Markopoulo K, Cleare AJ. The Maudsley Staging Method for Treatment Resistant Depression: prediction of longer-term outcome and persistence of symptoms. J Clin Psychiatry 2009; 70: 952-9.

15 Li C-T, Bai Y-M, Huang Y-L, Chen Y-S, Chen T-J, Cheng J-Y, et al. Association between antidepressant resistance in unipolar depression and subsequent bipolar disorder: cohort study. Br J Psychiatry 2012; 200: 45-51.

16. Wooderson SC, Juruena MF, Fekadu A, Commane C, Donaldson C, Cowan M et al. Prospective evaluation of specialist inpatient treatment for refractory affective disorders. J Affect Disord 2011; 131: 92-103.

17 Frank E, Prien RF, Jarrett RB, Keller MB, Kupfer DJ, Lavori PW, et al. Conceptualization and rationale for consensus definitions of terms in major depressive disorder. Remission, recovery, relapse, and recurrence. Arch Gen Psychiatry 1991; 48: 851-55.

18 Keller MB. Past, present, and future directions for defining optimal treatmen outcome in depression: remission and beyond. JAMA 2003; 289: 3152-60.

19 Coryell W, Endicott J, Keller M. Outcome of patients with chronic affective disorder: a five-year follow-up. Am J Psychiatry 1990; 147: 1627-33.

20 World Health Organization. The ICD-10 Classification of Mental and Behavioural Disorders: Clinical Descriptions and Diagnostic Guidelines. WHO, 1992.

21 American Psychiatric Association. Diagnostic and Statistical Manual of Mental Disorders (4th edn) (DSM-IV). APA, 1994.

22 Kennedy N, Abbott R, Paykel ES. Remission and recurrence of depression in the maintenance era: long-term outcome in a Cambridge cohort. Psychol Med 2003; 33: 827-38.

23 Kennedy N, Paykel ES. Residual symptoms at remission from depression: impact on long-term outcome. J Affect Disord 2004; 80: 135-44.
24 Beck AT, Ward $\mathrm{CH}$, Mendelson M, Mock J, Erbaugh J. An inventory for measuring depression. Arch Gen Psychiatry 1961; 4: 561-71.

25 Hamilton M. A rating scale for depression. J Neurol Neurosurg Psychiatry 1960; 23: 56-62.

26 Ware JJ, Kosinski M, Keller SD. A 12-Item Short-Form Health Survey: construction of scales and preliminary tests of reliability and validity. Med Care 1996; 34: 220-33.

27 Dalgard OS, Dowrick C, Lehtinen V, Vazquez-Barquero JL, Casey $\mathrm{P}$ Wilkinson G, et al. Negative life events, social support and gender difference in derpession: a multinational community survey with data from the ODIN study. Soc Psychiatry Psychiatr Epidemiol 2006; 41: 444-51.

28 Brugha T, Bebbington P, Tennant C, Hurry J. The List of Threatening Experiences: a subset of 12 life event categories with considerable long-term contextual threat. Psychol Med 1985; 15: 189-94.

29 Taylor D, Paton C, Kapur S. The Maudsley Prescribing Guidelines, 10th Edition. Informa Healthcare, 2009.

30 Dunner DL, Rush AJ, Russell JM, Burke M, Wooodard S, Wingard P, et al Prospective, long-term, multicentre study of the naturalistic outcomes of patients with treatment-resistant depression. J Clin Psychiatry 2006; 67: 688-95

31 Scott J. Chronic depression. Br J Psychiatry 1988; 153: 287-97.

32 Sackeim HA. The definition and meaning of treatment-resistant depression. J Clin Psychiatry 2001; 62 (suppl 16): 10-7.

33 British Medical Association, Royal Pharmaceutical Society. British National Formulary 63 (March 2012). Pharmaceutical Press, 2012.

34 Shergill SS, Robertson MM, Stein G, Bernadt M, Katona CL. Outcome in refractory depression. J Affect Disord 1999; 54: 287-94.

35 National Collaborating Centre for Mental Health. Depression. The Treatment and Management of Depression in Adults (Updated Edition) (Clinical Guideline CG90). British Psychological Society \& The Royal College of Psychiatrists, 2010.

36 Nierenberg AA, Price LH, Charney DS, Heninger GR. After lithium augmentation: a retrospective follow-up of patients with antidepressantrefractory depression. J Affect Disord 1990; 18: 167-75.

37 Leskela $U$, Rytsala $H$, Komulainen E, Melartin T, Sokero $P$, Lestela-Mielonen $\mathrm{P}$, et al. The influence of adversity and perceived social support on the outcome of major depressive disorder in subjects with different levels of depressive symptoms. Psychol Med 2006; 36: 779-88.

38 Meyers BS, Sirey JA, Bruce M, Hamilton M, Raue P, Friedman SJ, et al. Predictors of early recovery from major depression among persons admitted to community-based clinics: an observational study. Arch Gen Psychiatry 2002; 59: 729-35.

39 Nasser EH, Overholser JC. Recovery from major depression: the role of support from family, friends, and spiritual beliefs. Acta Psychiatr Scand 2005; 111: $125-32$.

40 Pare $\mathrm{CM}$. The present status of monoamine oxidase inhibitors. Br J Psychiatry 1985; 146: 576-84.

41 Tyrer $\mathrm{P}$. Towards rational therapy with monoamine oxidase inhibitors. Br J Psychiatry 1976; 128: 354-60.

42 Akiskal HS. Mood disorders: clinical features. In Kaplan \& Sadock's Comprehensive Textbook of Psychiatry, Ninth Edition (eds BJ Sadock, VA Sadock, P Ruiz): 1693-731. Lippincott Williams \& Wilkins, 2009. 